Also available at http://amc.imfm.si

ISSN 1855-3966 (printed edn.), ISSN 1855-3974 (electronic edn.)

ARS MATHEMATICA CONTEMPORANEA 5 (2012) 371-382

\title{
Two atlases of abstract chiral polytopes for small groups
}

\author{
Michael I. Hartley \\ DownUnder GeoSolutions, 76 Kings Park Rd, West Perth, 6005, Australia \\ Isabel Hubard \\ Instituto de Matemáticas, Universidad Nacional Autónoma de México \\ Ciudad Universitaria, México D.F. 04510 \\ Dimitri Leemans \\ Department of Mathematics, University of Auckland \\ Private Bag 92019, Auckland 1142, New Zealand
}

Received 28 February 2011, accepted 24 February 2012, published online 8 June 2012

\begin{abstract}
We construct chiral abstract polytopes in two different ways. Firstly we seek them as quotients of regular polytopes arising from the Atlas of Small Regular Polytopes (http: //www.abstract-polytopes.com/atlas/); the resulting atlas of chiral polytopes atlas is available on the website http://www.abstract-polytopes.com/ chiral/. Secondly, for each almost simple group $\Gamma$ such that $S \leq \Gamma \leq \operatorname{Aut}(S)$ where $S$ is a simple group and $\Gamma$ is a group of order less than 900,000 listed in the Atlas of Finite Groups, we give, up to isomorphism, the number of abstract chiral polytopes on which $\Gamma$ acts regularly. The results have been obtained using a series of MAGMA programs. All these polytopes are made available on the third author's website, at http: //math.auckland.ac.nz/ dleemans/CHIRAL/.
\end{abstract}

Keywords: Abstract chiral polytopes, almost simple groups.

Math. Subj. Class.: 52B15, 20F65

E-mail addresses: mikeh@dugeo.com (Michael I. Hartley), hubard@matem.unam.mx (Isabel Hubard), d.leemans@auckland.ac.nz (Dimitri Leemans) 


\section{Introduction}

Abstract polytopes are combinatorial structures that generalize the face lattice of convex polytopes. Abstract polytopes of rank 3 are essentially maps (2-embeddings of maps on surfaces); while every rank 3 polytope is indeed a map, the converse is not true; however, checking if a map is indeed a polytope or not is not a difficult task (it amounts to checking the so-called diamond condition for polytopes, defined in Section 2).

There are several approaches to classifying maps (and hypermaps) with high degree of symmetry. For instance, one can classify all regular or chiral maps of a given genus. Atlases of that kind have been built essentially by Conder and various collaborators $([2,3])$. One could also classify maps by their underlying graphs (see for example $[6,7,8,15]$ ) or by their automorphism groups.

The latter approach has also been used to classify regular abstract polytopes. There are three atlases of regular polytopes; all of them classify the polytopes by their automorphism groups. The first of these atlases, [11], contains information about all regular polytopes with automorphism group of size $n$, where $n$ is at most 2000, and not equal to 1024 or 1536. The second atlas, [18], contains all regular polytopes whose automorphism group is an almost simple group $\Gamma$ such that $S \leq \Gamma \leq \operatorname{Aut}(S)$, where $S$ is a simple group and $\Gamma$ is a group of order less than 1 million appearing in the Atlas of Finite Groups ([4]). The third atlas [14] extends the second atlas to sporadic groups.

The aim of this paper is to produce atlases of chiral abstract polytopes. In building these atlases we take two different approaches. The results of each appear in different websites. On the one hand, we find all the chiral polytopes for which the minimal regular cover falls into the Atlas of Small Regular Polytopes [11]. In particular, the minimal regular cover of all such chiral polytopes has at most 2,000 flags. On the other hand, we find chiral polytopes with automorphism group isomorphic to a small almost simple group, in the spirit of the second atlas described above.

The paper is organized as follows. Section 2 reviews the basics of abstract polytopes. In Section 3, we give the basics of abstract chiral polytopes. We also explain how to get chiral polytopes as quotients of regular polytopes. In Section 4 , we describe the information available on the two websites mentioned in the abstract.

\section{Basic notions}

We start by reviewing the basic theory of abstract polytopes and regular polytopes. For details, we refer the reader to [19].

An (abstract) polytope of rank $n$ or an $n$-polytope is a partially ordered set $\mathcal{P}$ endowed with a strictly monotone rank function having range $\{-1, \ldots, n\}$. The elements of $\mathcal{P}$ are called faces. For $0 \leq j<n$, a face of rank $j$ is often called a $j$-face and the faces of rank 0,1 and $n-1$ are usually called the vertices, edges and facets of the polytope, respectively. We shall ask that $\mathcal{P}$ have a smallest face $F_{-1}$, and a greatest face $F_{n}$ (called the improper faces of $\mathcal{P}$ ), and that each flag (that is, maximal chain of the order) of $\mathcal{P}$ contain exactly $n+2$ faces. Two flags are said to be adjacent if they differ by exactly one face, they are $j$-adjacent, if the rank of the face they differ on is precisely $j$. We also require that $\mathcal{P}$ be strongly flag-connected, that is, any two flags $\Phi, \Psi \in \mathcal{F}(\mathcal{P})$ can be joined by a sequence of flags $\Phi=\Phi_{0}, \Phi_{1}, \ldots, \Phi_{k}=\Psi$ such that each two successive flags $\Phi_{i-1}$ and $\Phi_{i}$ are adjacent with $\Phi \cap \Psi \subseteq \Phi_{i}$ for all $i$. Finally, we require the diamond condition, namely, whenever $F \leq G$, with $\operatorname{rank}(F)=j-1$ and $\operatorname{rank}(G)=j+1$, there are exactly two faces 
$H$ of rank $j$ such that $F \leq H \leq G$.

The diamond condition implies that given a flag $\Phi$ of $\mathcal{P}$, for each $i \in\{0, \ldots, n-1\}$ there exists a unique flag $\Phi^{i}$ which is $i$-adjacent flag to $\Phi$.

Given two faces $F$ and $G$ of a polytope $\mathcal{P}$ such that $F \leq G$, the section $G / F$ of $\mathcal{P}$ is the set of faces $\{H \in \mathcal{P} \mid F \leq H \leq G\}$, with the induced partial order. If $F_{0}$ is a vertex, then the section $F_{n} / F_{0}$ is called the vertex-figure of $F_{0}$. Note that every section $G / F$ of a polytope $\mathcal{P}$ is also a polytope and that $\operatorname{rank}(G / F)=\operatorname{rank}(G)-\operatorname{rank}(F)-1$.

Let $\mathcal{P}$ and $\mathcal{Q}$ be two $n$-polytopes. An isomorphism from $\mathcal{P}$ to $\mathcal{Q}$ is a bijection $\gamma: \mathcal{P} \rightarrow$ $\mathcal{Q}$ such that $\gamma$ and $\gamma^{-1}$ preserve the order. An anti-isomorphism $\delta: \mathcal{P} \rightarrow \mathcal{Q}$ is a bijection reversing the order, in which case $\mathcal{P}$ and $\mathcal{Q}$ are said to be duals of each other, and the usual convention is to denote $\mathcal{Q}$ by $\mathcal{P}^{*}$. (Note that $\left(\mathcal{P}^{*}\right)^{*} \cong \mathcal{P}$.) An isomorphism from $\mathcal{P}$ onto itself is called an automorphism of $\mathcal{P}$. The set of all automorphisms of $\mathcal{P}$ forms a group, its automorphism group, denoted by $\Gamma(\mathcal{P})$. It is not difficult to see that $\Gamma(\mathcal{P})$ acts freely on $\mathcal{F}(\mathcal{P})$, the set of all flags of $\mathcal{P}$. An anti-isomorphism from $\mathcal{P}$ to itself is called a duality. When a duality of $\mathcal{P}$ exists, $\mathcal{P}$ is said to be self-dual. Note that the set of all dualities is not a group, as the product of two dualities is in fact an automorphism. However, the dualities and automorphisms of $\mathcal{P}$ together do form the extended group of $\mathcal{P}$, denoted by $\bar{\Gamma}(\mathcal{P})$.

A polytope $\mathcal{P}$ is said to be regular if $\Gamma(\mathcal{P})$ is transitive on the flags of $\mathcal{P}$. The automorphism group of a regular polytope $\mathcal{P}$ is generated by $n$ involutions $\rho_{0}, \rho_{1}, \ldots, \rho_{n-1}$, such that each $\rho_{i}$ maps a given (base) flag $\Phi$ to the $i$-adjacent flag, $\Phi^{i}$. These distinguished generators satisfy (among others) the relations

$$
\left(\rho_{i} \rho_{j}\right)^{p_{i j}}=\epsilon \text { for } 0 \leq i \leq j \leq n-1
$$

where the symbol $\epsilon$ denotes the identity element of $\Gamma(\mathcal{P}), p_{i i}=1$ for all $i$, and $p_{j i}=p_{i j} \geq$ 2 whenever $|i-j|=1$, and $p_{i j}=2$ otherwise. Letting $p_{i}=p_{i-1, i}=p_{i, i-1}$ for $1 \leq i<n$, we say that $\mathcal{P}$ has Schläfli type $\left\{p_{1}, \ldots, p_{n-1}\right\}$.

Furthermore, the generators $\rho_{i}$ for $\Gamma(\mathcal{P})$ satisfy an additional condition, often called the intersection property, namely

$$
\left\langle\rho_{i} \mid i \in I\right\rangle \cap\left\langle\rho_{i} \mid i \in J\right\rangle=\left\langle\rho_{i} \mid i \in I \cap J\right\rangle \text { for every } I, J \subseteq\{0,1, \ldots, n-1\} .
$$

Conversely, if $\Gamma$ is a group generated by elements $\rho_{0}, \rho_{1}, \ldots, \rho_{n-1}$ which satisfy the relations (2.1) and condition (2.2), then there exists a polytope $\mathcal{P}$ with $\Gamma(\mathcal{P}) \cong \Gamma$. For more details on this correspondence, we refer to [19].

\section{Chiral polytopes}

In this section we define the basic properties of chiral polytopes. We state some relations between chiral and regular polytopes. For details see [20] and [21]. Finally, in a subsection, we explain how to get chiral polytopes as quotients of regular polytopes.

Every regular polytope $\mathcal{P}$ has a rotation subgroup $\Gamma^{+}(\mathcal{P})$ of $\Gamma(\mathcal{P})$ generated by

$$
\sigma_{i}:=\rho_{i-1} \rho_{i}, \quad i=1,2, \ldots, n-1 .
$$

These $\sigma_{i}$ satisfy at least the relations

$$
\begin{aligned}
\sigma_{i}^{p_{i}} & =\epsilon \quad \text { for } 1 \leq i \leq n-1 \\
\left(\sigma_{i} \sigma_{i+1} \ldots \sigma_{j}\right)^{2} & =\epsilon \quad \text { for } 1 \leq i<j \leq n-1 .
\end{aligned}
$$


Here again $\left\{p_{1}, p_{2}, \ldots, p_{n-1}\right\}$ is the Schläfli type of $\mathcal{P}$. Note that $\Gamma^{+}(\mathcal{P})$ has index at most two in $\Gamma(\mathcal{P})$. A regular $n$-polytope $\mathcal{P}$ is called directly or orientably regular if $\Gamma^{+}(\mathcal{P})$ has index two in $\Gamma(\mathcal{P})$.

An $n$-polytope $\mathcal{P}$ with base flag $\Phi$ is called chiral if it is not regular, but there exist automorphisms $\sigma_{1}, \sigma_{2}, \ldots, \sigma_{n-1}$ such that each $\sigma_{i}$ fixes all faces in $\Phi$ different from $(\Phi)_{i-1}$ and $(\Phi)_{i}$, and cyclically permutes consecutive $i$-faces of $\mathcal{P}$ in the rank 2 section $(\Phi)_{i+1} /(\Phi)_{i-2}$ of $\mathcal{P}$. (By $(\Phi)_{i}$ we mean here the $i$-face of $\Phi$.) Such automorphisms generate $\Gamma(\mathcal{P})$ and are called the distinguished generators of $\Gamma(\mathcal{P})$ with respect to $\Phi$. These distinguished generators satisfy the relations (3.1) and (3.2), and $\left\{p_{1}, \ldots, p_{n-1}\right\}$ is again said to be the Schläfli type of $\mathcal{P}$. Note that chiral polytopes occur in pairs of enantiomorphic forms, with one being the 'mirror image' of the other. In fact, one enantiomorphic form of a polytope is associated with a base flag $\Phi$ and the other with any of the flags adjacent to it. Furthermore, if $\sigma_{1}, \ldots, \sigma_{n-1}$ are the distinguished generators of a chiral polytope $\mathcal{P}$ with respect to a base flag $\Phi$, then the distinguished generators of $\mathcal{P}$ with respect to $\Phi^{0}$ (i.e. the enantiomorphic form) are $\sigma_{1}^{-1}, \sigma_{1}^{2} \sigma_{2}, \sigma_{3}, \ldots, \sigma_{n-1}$.

In a similar way as for the regular case, the distinguished generators of the automorphism group of a chiral polytope satisfy an intersection condition, arising from considering the stabilizers of the chains $\Phi_{\bar{J}}:=\left\{(\Phi)_{j} \mid j \notin J\right\}$, for each $J \subseteq\{0,1, \ldots, n-1\}$.

In order to state this intersection condition we first define the "half-turns" in $\Gamma(\mathcal{P})$ to be the involutions $\tau_{i, j}:=\sigma_{i} \ldots \sigma_{j}$, for $1 \leq i<j \leq n-1$. Furthermore, for each $i \in\{1, \ldots n-1\}$, we define $\tau_{i, i}:=\sigma_{i}$ and $\tau_{0, j}=\tau_{i, n}:=\epsilon$, the identity element of $\Gamma(\mathcal{P})$. Then the stabilizer in $\Gamma(\mathcal{P})$ of the chain $\Phi_{\bar{J}}$ (with $J \subseteq\{0, \ldots, n-1\}$ ) is the subgroup

$$
\Gamma_{J}:=\left\langle\tau_{i, j} \mid i \leq j ; i-1, j \in J\right\rangle .
$$

Hence, the intersection condition for $\Gamma$, stated in terms of these half-turns, is given by

$$
\Gamma_{I} \cap \Gamma_{J}=\Gamma_{I \cap J}, \quad \text { for all } I, J \subseteq\{0, \ldots, n-1\} .
$$

Conversely, if $\Gamma$ is any group generated by elements $\sigma_{1}, \sigma_{2}, \ldots, \sigma_{n-1}$ which satisfy the relations (3.1) and (3.2), as well as the intersection condition (3.3), then there exists a polytope $\mathcal{P}$ of rank $n$ which is either directly regular or chiral. The Schläfli type of $\mathcal{P}$ is $\left\{p_{1}, \ldots, p_{n-1}\right\}$, where $p_{i}$ is the order of $\sigma_{i}$ (for $\left.1 \leq i<n\right)$; and $\Gamma(\mathcal{P}) \cong \Gamma$ if $\mathcal{P}$ is chiral, or $\Gamma^{+}(\mathcal{P}) \cong \Gamma$ if $\mathcal{P}$ is directly regular.

Moreover, $\mathcal{P}$ is directly regular if and only if there exists an involutory group automorphism $\rho: \Gamma \rightarrow \Gamma$ such that

$$
\rho\left(\sigma_{1}\right)=\sigma_{1}^{-1}, \rho\left(\sigma_{2}\right)=\sigma_{1}^{2} \sigma_{2}, \rho\left(\sigma_{i}\right)=\sigma_{i} \text { for } 3 \leq i \leq n-1
$$

(or in other words, acting like conjugation by the generator $\rho_{0}$ in the orientably regular case). That is, $\mathcal{P}$ is chiral whenever no such automorphism exists.

Hence, to know whether or not a given a group $\Gamma$ is the automorphism group of a chiral polytope, one would have to check if $\Gamma$ can be generated by elements $\sigma_{1}, \ldots, \sigma_{n-1}$ satisfying relations (3.1) and (3.2), as well as the intersection condition (3.3). In addition one would have to check that there exists no group automorphism as in (3.4).

A self-dual polytope $\mathcal{P}$ is said to be properly self-dual if there exists a duality $\delta$ of $\mathcal{P}$ mapping a base flag to a flag in the same orbit. Clearly, $\delta$ must preserve the flag orbits. If no such $\delta$ exits, we say that $\mathcal{P}$ is improperly self-dual. Hence if $\mathcal{P}$ is a properly (improperly) self-dual chiral polytope, then every duality of $\mathcal{P}$ preserves (interchanges) the two flag orbits. 


\subsection{Chiral polytopes as quotients of regular ones}

Another way to identify chiral polytopes is to seek them as quotients of regular covering polytopes. In [9] it is shown that any polytope $\mathcal{Q}$ may be written in the form $\mathcal{P} / \mathcal{N}$ for some regular polytope $\mathcal{P}$ and some subgroup $\mathcal{N}$ of the automorphism group of $\mathcal{P}$. These subgroups $\mathcal{N}$ of $\Gamma(\mathcal{P})=\left\langle\rho_{0}, \ldots, \rho_{n-1}\right\rangle$ satisfy a rather technical condition, and are called semisparse subgroups. The automorphism group of $\mathcal{Q}$ may be written (see [10]) as $N_{\Gamma(\mathcal{P})}(\mathcal{N}) / \mathcal{N}$, where $N_{\Gamma(\mathcal{P})}(\mathcal{N})$ is the normaliser of $\mathcal{N}$ in $\Gamma(\mathcal{P})$. Note that the number of flags of $\mathcal{Q}$ is $|\Gamma(\mathcal{P}): \mathcal{N}|$. If we are dealing with finite groups, we can conclude immediately from the definition of a chiral polytope :

Theorem 3.1. The polytope $\mathcal{Q}=\mathcal{P} / \mathcal{N}$ is chiral if and only if $N_{\Gamma(\mathcal{P})}(\mathcal{N})$ has index 2 in $\Gamma(\mathcal{P})$, and contains none of the generators $\rho_{0}, \ldots, \rho_{n-1}$.

This suggests an algorithm for searching for chiral quotients of a given regular polytope $\mathcal{P}$ with automorphism group $\Gamma(\mathcal{P})=\left\langle\rho_{0}, \ldots, \rho_{n-1}\right\rangle$.

- Find all normal subgroups $G$ of $\Gamma(\mathcal{P})$ of index 2 which do not contain any of the $\rho_{i}$.

- For each such group $G$, find all the normal subgroups $\mathcal{N}$ of $G$ which are not normal in $\Gamma(\mathcal{P})$. This is sufficient and necessary to ensure that $G$ is the normaliser for $\mathcal{N}$ in $\Gamma(\mathcal{P})$.

- Ignore any such $\mathcal{N}$ that are not semisparse in $\Gamma(\mathcal{P})$. This ensures that we only retain chiral polytopes, and ignore other combinatorial structures.

- If it is desired to find only chiral quotients $\mathcal{Q}$ of $\mathcal{P}$ for which $\mathcal{P}$ is the minimal covering polytope whose automorphism group acts on $\mathcal{Q}$ via the flag action, then ignore any such $\mathcal{N}$ for which $\operatorname{Core}_{\Gamma(\mathcal{P})}(\mathcal{N})=\cap_{w \in \Gamma(\mathcal{P})} \mathcal{N}^{w}$ is not the trivial group.

- Any $\mathcal{N}$ still retained will be such that $\mathcal{P} / \mathcal{N}$ is a chiral polytope $\mathcal{Q}$ with automorphism group $G / \mathcal{N}$. In fact, this algorithm produces exactly two such $\mathcal{N}$ for each $\mathcal{Q}$, since it finds separately $\mathcal{N}$ and $\mathcal{N}^{\rho_{0}}$ for each $G$. These duplicates are easy to identify and remove from the list.

\section{The Atlases}

As we mentioned before, we constructed two different atlases, using different approaches.

One atlas, The Atlas Of Chiral Polytopes With Small Regular Covers [12], contains information about all chiral polytopes whose regular covers have automorphism group of order at most 2000, but not 1024 or 1536 . To construct it, the algorithm described in Section 3.1 was tried on every polytope in the Atlas of Small Regular Polytopes [11].

The other atlas, The Atlas of Chiral polytopes for Small Almost Simple Groups [13], was built by writing MAGMA [1] programs that classify, up to isomorphism, ordered tuples of generators of a given group $\Gamma$ that satisfy conditions (3.1), (3.2) and (3.3). Our program then tells us whether such generators correspond to chiral or orientably regular polytopes. This program was run on all almost simple groups $\Gamma$ such that $S \leq \Gamma \leq \operatorname{Aut}(S)$ where $S$ is a simple group appearing in the Atlas of Finite Groups by Conway et al [4] and $\Gamma$ is of order less than 1 million .

The groups analysed are subdivided into six families, namely

- Sporadic groups and their automorphism groups; 


\begin{tabular}{|c|c|c|}
\hline $\mathcal{P}$ & $\operatorname{Aut}(\mathcal{P})$ & $\operatorname{Aut}(\mathcal{Q})$ \\
\hline \hline$\{3,3,8\} * 768 b$ & $\left(\left(Q_{8} \times 2\right) \rtimes 2\right) \rtimes S_{4}$ & $Q_{8} \rtimes S_{4}$ \\
$\{3,6,9\} * 972 a$ & $3^{3} \rtimes\left(D_{9} \times 2\right)$ & $\left(3^{3} \rtimes 3\right) \rtimes 2$ \\
$\{3,6,18\} * 1944 a$ & $\left(3^{3} \rtimes\left(D_{9} \times 2\right)\right) \times 2$ & $\left(\left(3^{3} \rtimes 3\right) \rtimes 2\right) \times 2$ \\
$\{6,6,9\} * 1944 a$ & $\left(3^{3} \rtimes\left(D_{9} \times 2\right)\right) \times 2$ & $\left(\left(3^{3} \rtimes 3\right) \rtimes 2\right) \times 2$ \\
\hline
\end{tabular}

Table 1: Rank 4 chiral quotients $\mathcal{Q}$ of small regular polytopes

- Alternating groups and their automorphism groups;

- $\operatorname{PSL}(2, q)$ groups and their automorphism groups;

- Other linear groups and their automorphism groups;

- Unitary groups and their automorphism groups;

- Suzuki groups and their automorphism groups.

\section{Results}

As expected, the first atlas of chiral polytopes, those obtained as quotient of regular polytopes, gave us fewer examples of chiral polytopes that the one built with almost simple groups.

In the first atlas, in total, 56 chiral polytopes were discovered, 48 of rank 3 , and 8 of rank 4 . Note that a polytope and its dual are counted as two polytopes in these totals. One of each dual pair of the rank 4 polytopes is outlined in Table 1, and the entire collection may be perused online (see [12]).

The polytopes in the last two rows of this table do indeed have isomorphic rotation groups and isomorphic automorphism groups for their regular covers. The vertex figures of type $\{6,9\}$ and $\{6,18\}$ are in turn chiral and appear on the website.

The results of the Atlas of Small Chiral Polytopes for Small Almost Simple Groups are summarised in the Tables 2 to 7. The Tables are all organised as follows. For a group $G$, we give its automorphism group $A u t(G)$, its order (\#G), the number of polytopes that $G$ acts on regularly up to isomorphism, and the number of polytopes $G$ acts on chirally up to isomorphism. These latter two numbers are sometimes split in several numbers. When we write $x=x_{1}+x_{2}+\ldots+x_{n}$, it means there are $x_{1}$ (resp. $x_{2}, \ldots x_{n}$ ) polytopes of rank 3 (resp. $4, \ldots, n+2$ ). Otherwise, it means all polytopes found are of rank three.

On the website, for each group, a list of all polytopes found is available, sorted by rank and by Schläfli symbols, as well as a MAGMA file containing the involutions generating the corresponding groups.

\section{Some observations on the results}

One of the reasons to build such atlases is to try to get insight into the question of whether are more or fewer regular polytopes than chiral polytopes. Of course, the answer to this question may depend on tones measure.

For instance, in Marston Conder's website (http://www . math. auckland.ac . $\mathrm{nz} / \sim \mathrm{conder} /$ ), we find maps classified by genus. It turns out that there are 3378 orientable regular maps of genus less than 102, 862 non-orientable regular maps of genus less 


\begin{tabular}{|c|c|c|c|c|}
\hline $\mathbf{G}$ & Aut(G) & \#G & \#Regular & \#Chiral \\
\hline \hline$M_{11}$ & $M_{11}$ & 7920 & 0 & 66 \\
$M_{12}$ & $M_{12}: 2$ & 95040 & $67=40+27$ & $184=118+64+2$ \\
$M_{12}: 2$ & $M_{12}: 2$ & 190080 & $502=416+86$ & $700=608+92$ \\
$J_{1}$ & $J_{1}$ & 175560 & $300=296+4$ & $1096=1056+40$ \\
$M_{22}$ & $M_{22}: 2$ & 443510 & 0 & 242 \\
$M_{22}: 2$ & $M_{22}: 2$ & 887040 & $375=252+123$ & $1506=1442+64$ \\
$J_{2}$ & $J_{2}: 2$ & 604800 & $292=261+31$ & $986=888+98$ \\
\hline & & Total & 1536 & 4780 \\
\hline
\end{tabular}

Table 2: Sporadic groups and their automorphism groups

than 203 and 594 chiral orientably-regular maps of genus less than 102. So in terms of (small) genus, it seems that maps are more often regular than chiral.

Here we measure our results in terms of number of polytopes, up to isomorphism, for a given group. We say that a group is more chiral than regular if it has more chiral polytopes than regular polytopes. The Atlas of Regular Polytopes for Small Groups [18] gave, up to isomorphism and duality, 5265 regular polytopes in total. We computed here that there are, up to isomorphism, 9205 such polytopes, when $\operatorname{Aut}\left(J_{2}\right)$ is not taken into account. We found, up to isomorphism, 17114 chiral polytopes in this atlas. Of course, chiral polytopes come in pairs of enantiomorphic forms. Therefore, if we decide to count one chiral polytope and its enantiomorphic form as one, we get here $17114 / 2=8557$ polytopes. What clearly appears from Tables 2 to 7 is that some families of almost simple groups are more chiral than others. For instance, for sporadic groups, we get 4780 chiral polytopes, against 1536 regular polytopes. On the other hand, we get 2050 chiral polytopes and 3904 regular polytopes for almost simple groups of $P S L(2, q)$ type. But for the latter family, there are 506 chiral polytopes of rank at least 4 and only 51 regular polytopes of rank at least 4 . Unitary groups and Suzuki groups also seem to be much more chiral than regular.

\section{Acknowledgements}

We gratefully acknowledge financial support of the "Fonds David et Alice Van Buuren", the "Communauté Française de Belgique, Action de Recherche Concertée", and "IACOD - UNAM (Grant IA101911) Mexico" for this project. We also thank Marston Conder for interesting discussions during the writing of this paper.

\section{References}

[1] W. Bosma, C. Cannon and C. Playoust, The Magma algebra system I: The user language, $J$. Symbolic Comput. 24 (1997), 235-265.

[2] M. Conder, Regular maps and hypermaps of Euler characteristic -1 to -200 , J. Combin. Theory Ser. B 99 (2009), 455-459.

[3] M. Conder and P. Dobcsanyi, Determination of all regular maps of small genus, J. Combin. Theory Ser. B 81 (2001), 224-242.

[4] J. H. Conway, R. T. Curtis, S. P. Norton, R. A. Parker and R.A. Wilson, Atlas of Finite Groups, Oxford U. P., Oxford, 1985. 


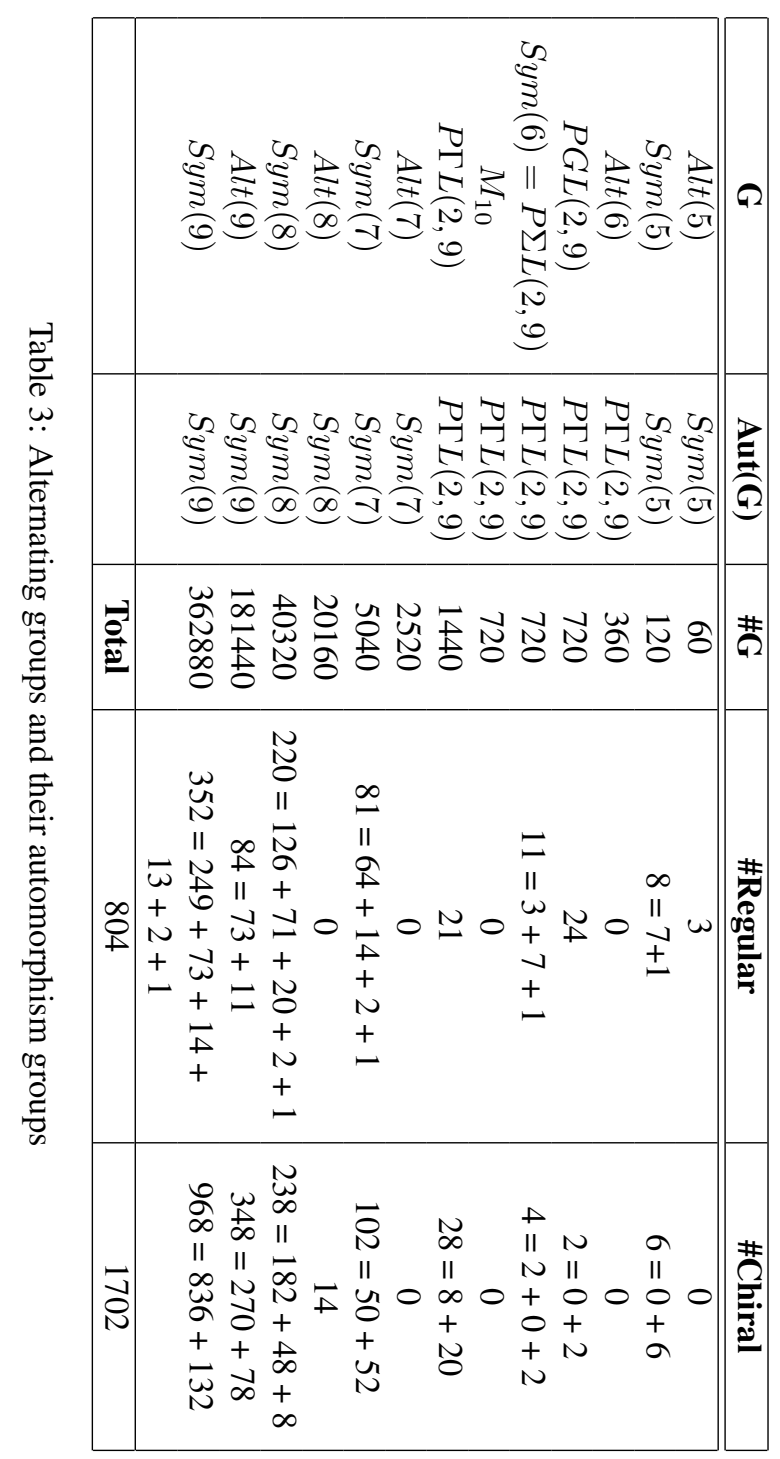




\begin{tabular}{|c|c|c|c|c|}
\hline $\mathbf{G}$ & $\operatorname{Aut}(\mathbf{G})$ & \#G & \#Regular & \#Chiral \\
\hline $\operatorname{Alt}(5)=P S L(2,4)=P S L(2,5)$ & $\operatorname{Sym}(5)$ & 60 & 3 & 0 \\
\hline $\operatorname{Sym}(5)=P G L(2,5)$ & $\operatorname{Sym}(5)$ & 120 & $8=7+1$ & $6=0+6$ \\
\hline$P S L(3,2)=P S L(2,7)$ & $P \Gamma L(2,7)$ & 168 & 0 & 0 \\
\hline$P G L(2,7)=P \Gamma L(2,7)$ & $P \Gamma L(2,7)$ & 336 & 28 & $10=0+10$ \\
\hline $\operatorname{Alt}(6)=P S L(2,9)$ & $P \Gamma L(2,9)$ & 360 & 0 & 0 \\
\hline$P G L(2,9)$ & $P \Gamma L(2,9)$ & 720 & 24 & $2=0+2$ \\
\hline $\operatorname{Sym}(6)=P \Sigma L(2,9)$ & $P \Gamma L(2,9)$ & 720 & $11=3+7+1$ & $4=2+0+2$ \\
\hline$M_{10}$ & $P \Gamma L(2,9)$ & 720 & 0 & 0 \\
\hline$P \Gamma L(2,9)$ & $P \Gamma L(2,9)$ & 1440 & 21 & $28=8+20$ \\
\hline$P S L(2,8)$ & $P \Gamma L(2,8)$ & 504 & 14 & $2=0+2$ \\
\hline$P \Gamma L(2,8)$ & $P \Gamma L(2,8)$ & 1512 & 0 & 28 \\
\hline$P S L(2,11)$ & $P G L(2,11)$ & 660 & $5=4+1$ & 0 \\
\hline$P G L(2,11)$ & $P G L(2,11)$ & 1320 & 78 & $24=0+24$ \\
\hline $\operatorname{PSL}(2,13)$ & $P G L(2,13)$ & 1092 & 19 & $6=0+6$ \\
\hline$P G L(2,13)$ & $P G L(2,13)$ & 2184 & 111 & $14=0+14$ \\
\hline$P S L(2,17)$ & $P G L(2,17)$ & 2448 & 30 & $10=0+10$ \\
\hline$P G L(2,17)$ & $P G L(2,17)$ & 4896 & 208 & $8=0+8$ \\
\hline $\operatorname{PSL}(2,19)$ & $P G L(2,19)$ & 3420 & $31=30+1$ & $4=0+4$ \\
\hline$P G L(2,19)$ & $P G L(2,19)$ & 6840 & 268 & $28=0+28$ \\
\hline $\operatorname{PSL}(2,16)$ & $P \Gamma L(2,16)$ & 4080 & 51 & $2=0+2$ \\
\hline$P S L(2,16): 2$ & $P \Gamma L(2,16)$ & 8160 & $46=39+7$ & $48=32+16$ \\
\hline$P \Gamma L(2,16)$ & $P \Gamma L(2,16)$ & 16320 & 0 & 122 \\
\hline $\operatorname{PSL}(2,23)$ & $P G L(2,23)$ & 6072 & 52 & 0 \\
\hline$P G L(2,23)$ & $P G L(2,23)$ & 12144 & 408 & $10=0+10$ \\
\hline$P S L(2,25)$ & $P \Gamma L(2,25)$ & 7800 & 30 & $2=0+2$ \\
\hline$P G L(2,25)$ & $P \Gamma L(2,25)$ & 15600 & 240 & $6=0+6$ \\
\hline$P \Sigma L(2,25)$ & $P \Gamma L(2,25)$ & 15600 & $88=61+27$ & $62=38+24$ \\
\hline$P S L(2,25) .2$ & $P \Gamma L(2,25)$ & 7800 & 0 & 30 \\
\hline$P \Gamma L(2,25)$ & $P \Gamma L(2,25)$ & 31200 & 117 & $152=108+44$ \\
\hline$P S L(2,27)$ & $P \Gamma L(2,27)$ & 9828 & 27 & 0 \\
\hline$P G L(2,27)$ & $P \Gamma L(2,27)$ & 19656 & 190 & $4=0+4$ \\
\hline$P \Sigma L(2,27)$ & $P \Gamma L(2,27)$ & 29484 & 0 & 108 \\
\hline$P \Gamma L(2,27)$ & $P \Gamma L(2,27)$ & 58968 & 0 & 324 \\
\hline$P S L(2,29)$ & $P G L(2,29)$ & 12180 & 93 & $10=0+10$ \\
\hline$P G L(2,29)$ & $P G L(2,29)$ & 24360 & 655 & $26=0+26$ \\
\hline$P S L(2,31)$ & $P G L(2,31)$ & 14880 & 96 & $6=0+6$ \\
\hline$P G L(2,31)$ & $P G L(2,31)$ & 29760 & 766 & $46=0+46$ \\
\hline$P S L(2,32)$ & $P \Gamma L(2,32)$ & 32736 & 186 & $6=0+6$ \\
\hline \multirow[t]{2}{*}{$P \Gamma L(2,32)$} & $P \Gamma L(2,32)$ & 163680 & 0 & 744 \\
\hline & & Total & 3904 & 2050 \\
\hline
\end{tabular}

Table 4: $P S L(2, q)$ groups and their automorphism groups 


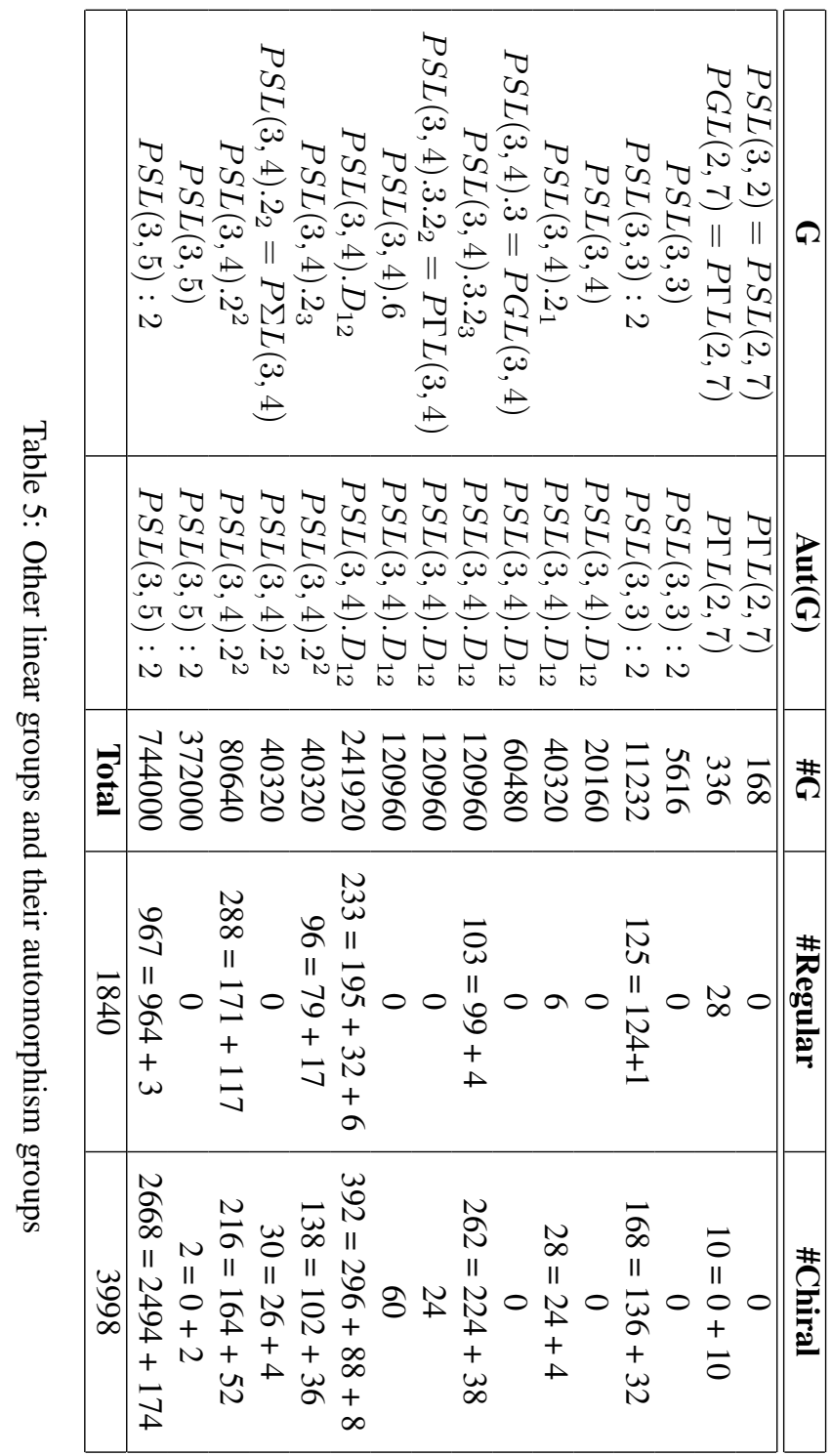




\begin{tabular}{|c|c|c|c|c|}
\hline $\mathbf{G}$ & $\operatorname{Aut}(G)$ & \#G & \#Regular & \#Chiral \\
\hline$P S U(3,3)$ & $P \Gamma L(3,3)$ & 6048 & 0 & 0 \\
\hline$P \Gamma U(3,3)$ & $P \Gamma U(3,3)$ & 12096 & $60=48+12$ & $166=146+20$ \\
\hline$P S U(4,2)$ & $P \Gamma U(4,2)$ & 25920 & 0 & 26 \\
\hline$P \Gamma U(4,2)$ & $P \Gamma U(4,2)$ & 51840 & $276=162+96+18$ & $370=270+100$ \\
\hline$P S U(3,4)$ & $P \Gamma U(3,4)$ & 62400 & 0 & 0 \\
\hline$P S U(3,4): 2$ & $P \Gamma U(3,4)$ & 124800 & $153=150+3$ & $418=376+42$ \\
\hline$P \Gamma U(3,4)$ & $P \Gamma U(3,4)$ & 249600 & 0 & 526 \\
\hline$P S U(3,5)$ & $P \Gamma U(3,5)$ & 126000 & 0 & 0 \\
\hline$P G U(3,5)$ & $P \Gamma U(3,5)$ & 378000 & 0 & 0 \\
\hline$P \Gamma U(3,5)$ & $P \Gamma U(3,5)$ & 756000 & $488=468+20$ & $1754=1580+174$ \\
\hline \multirow[t]{2}{*}{$P \Sigma U(3,5)$} & $P \Sigma U(3,5)$ & 252000 & $225=204+21$ & $962=834+120+8$ \\
\hline & & Total & 1202 & 4222 \\
\hline
\end{tabular}

Table 6: Unitary groups and their automorphism groups

\begin{tabular}{|c|c|c|c|c|}
\hline G & Aut(G) & \#G & \#Regular & \#Chiral \\
\hline \hline$S z(8)$ & $S z(8): 3$ & 29120 & 14 & 128 \\
$S z(8): 3$ & $S z(8): 3$ & 87360 & 0 & 284 \\
\hline & & Total & 14 & 412 \\
\hline
\end{tabular}

Table 7: Suzuki groups and their automorphism groups

[5] L. E. Dickson, Linear groups: With an exposition of the Galois field theory, Dover Publications Inc., New York, 1958.

[6] S. F. Du, J. H. Kwak and R. Nedela, A classification of regular embeddings of graphs of order a product of two primes, J. Algebraic Combin. 19 (2004), 123-141.

[7] S. F. Du, J. H. Kwak and R. Nedela, Regular embeddings of complete multipartite graphs. European J. Combin. 26 (2005), 505-519.

[8] A. Gardiner, R. Nedela, J. Širáň and M. Škoviera, Characterization of graphs which underlie regular maps on closed surfaces, J. London Math. Soc. 59 (1999), 100-108.

[9] M. I. Hartley, All Polytopes Are Quotients, and Isomorphic Polytopes are Quotients by Conjugate Subgroups, Disc. Comp. Geom 21 (1999), 289-298.

[10] M. I. Hartley, More On Quotient Polytopes. Aeq. Math. 57 (1999), 108-120.

[11] M. I. Hartley, An Atlas Of Small Regular Polytopes. Per. Math. Hung. 53 (2006),149-156, Full atlas online at http://www. abstract-polytopes.com/atlas/.

[12] M. I. Hartley, I. Hubard and D. Leemans, Atlas Of Chiral Polytopes With Small Regular Covers, http://www.abstract-polytopes.com/chiral/.

[13] M. I. Hartley, I. Hubard and D. Leemans, An Atlas of Chiral Polytopes for Small Almost Simple Groups, http://math. auckland.ac.nz/ dleemans/CHIRAL/.

[14] M. I. Hartley and A. Hulpke, Polytopes Derived from Sporadic Simple Groups, Contributions to Discrete Mathematics 5 (2010), 106-118, full atlas online at http://www . abstract-polytopes.com/sporpolys/. 
[15] L. D. James and G. A. Jones, Regular orientable embeddings of complete graphs, J. Combin. Theory Ser. B 39 (1985), 353-367.

[16] J. H. Kwak and Y. S. Kwon, Regular orientable embeddings of complete bipartite graphs, $J$. Graph Theory 50 (2005), 105-122.

[17] D. Leemans, Almost simple groups of Suzuki type acting on polytopes, Proc. Amer. Math. Soc. 134 (2006), 3649-3651.

[18] D. Leemans and L. Vauthier, An atlas of abstract regular polytopes for small groups, Aequationes Math. 72 (2006), 313-320, full atlas online at http://math. auckland.ac.nz/ \$ sim\$leemans/polytopes/.

[19] P. McMullen and E. Schulte, Abstract Regular Polytopes, Encyclopedia of Math. And its Applications. 92, Cambridge U. P., Cambridge, 2002.

[20] E. Schulte and A. Ivić Weiss, Chiral Polytopes, Applied Geometry and Discrete Mathematics (The Victor Klee Festschrift”), DIMACS Series in Discrete Matematics and Theoretical Computer Science 4 (1991), 493-516.

[21] E. Schulte and A. Ivić Weiss, Chirality and Projective linear groups. Discrete Math. 131 (1994), 221-261.

[22] D. Sjerve and M. Cherkassoff, On groups generated by three involutions, two of which commute, in: The Hilton Symposium 1993 (Montreal, PQ), volume 6 of CRM Proc. Lecture Notes, Amer. Math. Soc., Providence, RI, 1994, pp. 169-185. 